\title{
Spot-like structures of neutron star surface magnetic fields
}

\author{
U. Geppert ${ }^{1}$, M. Rheinhardt ${ }^{1}$, and J. Gil ${ }^{1,2}$ \\ 1 Astrophysikalisches Institut Potsdam, An der Sternwarte 16, 14482 Potsdam, Germany \\ 2 Institute of Astronomy, University of Zielona Góra, Lubuska 2, 65-265 Zielona Góra, Poland
}

Received 9 July 2003 / Accepted 3 November 2003

\begin{abstract}
There is growing evidence, based on both X-ray and radio observations of isolated neutron stars, that besides the large-scale (dipolar) magnetic field, which determines the pulsar spin-down behaviour, small-scale poloidal field components are present, which have surface strengths one to two orders of magnitude larger than the dipolar component. We argue in this paper that the Hall-effect can be an efficient process in producing such small-scale field structures just above the neutron star surface. It is shown that due to a Hall-drift induced instability, poloidal magnetic field structures can be generated from strong subsurface toroidal fields, which are the result of either a dynamo or a thermoelectric instability acting at early times of a neutron star's life. The geometrical structure of these small-scale surface anomalies of the magnetic field resembles that of some types of "star-spots". The magnetic field strength and the length-scales are comparable with values that can be derived from various observations.
\end{abstract}

Key words. stars: neutron - stars: pulsars - stars: magnetic fields

\section{Observational evidence}

There exists both observational and theoretical evidence of the existence of strong small-scale magnetic field structures in the surface layers of isolated neutron stars. More and more, it becomes clear that neutron stars of nearly all ages must possess magnetic field structures, which are much more complicated than the simple assumption of a star centered magnetic dipole suggests. The polar surface magnetic field strength of such a dipole is conventionally estimated by $B_{\mathrm{d}} \sim 6.4 \times 10^{19} \sqrt{P \dot{P}} \mathrm{G}$, where standard neutron star quantities are assumed and the rotational period $P$ (in seconds) and its temporal derivative $\dot{P}$ are taken from radio and/or X-ray timing observations.

Recently, Becker et al. (2003) reported the (marginal) detection of an electron cyclotron line at about $3.3 \mathrm{keV}$ in the Chandra-spectra of the millisecond pulsar B1821-24. The energetic location of that line corresponds to a surface magnetic field strength of $3 \times 10^{11} \mathrm{G}$, while $P$ and $\dot{P}$ yield a dipolar surface field strength of about $4.5 \times 10^{9} \mathrm{G}$, almost two orders of magnitude lower.

Haberl et al. (2003) analysed the XMM-spectra of the isolated neutron star RBS 1223 and found evidence for a proton cyclotron absorption line in the energy range of $0.1 \ldots 0.3 \mathrm{keV}$, which is consistent with a magnetic surface field strength of $(2 \ldots 6) \times 10^{13} \mathrm{G}$. While $P=10.31 \mathrm{~s}$ seems to be a well settled value for the rotational period, the determination of $\dot{P}$ is a much more delicate task in this case. Recent evaluations of XMM data indicate, however, that the global dipolar

Send offprint requests to: $\mathrm{U}$. Geppert, e-mail: urme@aip.de field attributed to $P$ and $\dot{P}$ is at least one order of magnitude smaller than the field corresponding to the proton cyclotron line (Schwope 2003, private communication). Likewise, for the pulsar 1E 1207.4-5209 a surface field of $\sim 1.5 \times 10^{14} \mathrm{G}$ has been estimated (Sanwal et al. 2002), whereas the estimate for its dipolar field is $(2 \ldots 4) \times 10^{12} \mathrm{G}$ (Pavlov et al. 2002).

These X-ray observations indicate that, apart from a largescale dipolar magnetic field which determines the spin-down behaviour of the pulsar, much stronger but short-ranged field components close to the neutron star surface exist. They do not affect significantly the braking of the star's rotation, but can affect the magnetospheric processes in the vicinity of the polar cap (defined by the totality of all open dipolar field lines).

It is commonly accepted that pulsar radio emission is generated within a dense electron-positron plasma, the creation of which requires an ultra-strong potential drop that accelerates charged particles along curved magnetic field lines. The observed phenomenon of drifting subpulses strongly suggests that this potential drop results from the deficiency in the actual charge density with respect to the so-called co-rotational charge density (Goldreich \& Julian 1969) just above the polar cap surface (see Gil et al. 2003). The formation of such a charge-depleted region (called "polar gap" after Ruderman \& Sutherland 1975, who proposed it for the first time) requires a strong and highly non-dipolar surface magnetic field, with radii of curvature much smaller than $10^{6} \mathrm{~cm}$ and magnitudes close to $10^{13} \mathrm{G}$, irrespective of the dipolar field strength inferable from the pulsar spin-down (Gil \& Mitra 2001; Gil \& Melikidze 2002; Gil et al. 2003). Moreover, several periodicities observed in the phenomenon of drifting subpulses strongly 
suggest that the subpulse-associated plasma filaments (called "sparks" after Ruderman \& Sutherland 1975) circulate around a local magnetic pole ${ }^{1}$ (Deshpande \& Rankin 1999, 2001; Gil $\&$ Sendyk 2003). Accordingly, the small-scale surface magnetic field anomalies are supposed to show spot-like structures allowing a persistent arrangement of drifting sparks (due to the well known $\boldsymbol{E} \times \boldsymbol{B}$ plasma drift mechanism) in the form of quasi-annular patterns (Gil \& Sendyk 2000; Fan et al. 2001).

\section{The Hall-drift induced instability}

It is well known that the Hall-effect, probably via a cascade (Goldreich \& Reisenegger 1992), causes the generation of smaller scaled magnetic field components out of an existing large scale field. This process has been discussed for neutron star magnetic fields by a number of authors (see, e.g., Shalybkov \& Urpin 1997; Vainshtein et al. 2000; Hollerbach \& Rüdiger 2002). Here, however, we want to refer to the fact that small-scale poloidal fields close to the neutron star surface can be generated from a subsurface toroidal magnetic field by a Hall-drift induced instability (HDI). The basic prerequisite for this instability is that a sufficiently strong and inhomogeneous background field exists (Rheinhardt \& Geppert 2002; Geppert \& Rheinhardt 2002; Rheinhardt et al. 2003, RKG03). We demonstrate, that strength and spatial structure of the HDI modes resulting from a realistic NS crust model are consistent with quantities derived from observations.

The decay of a magnetic field in the almost crystallised crust of a NS is governed by

$$
\begin{aligned}
& \dot{\boldsymbol{B}}=-\operatorname{curl}(\eta \operatorname{curl} \boldsymbol{B}+\alpha(\operatorname{curl} \boldsymbol{B} \times \boldsymbol{B})) \\
& \operatorname{div} \boldsymbol{B}=0,
\end{aligned}
$$

where the diffusivity $\eta$ and the Hall-parameter $\alpha$ determine the intensity of Ohmic decay and Hall-drift, respectively, in their spatial distribution. Linearization of Eq. (1) with respect to a background field $\boldsymbol{B}_{0}$ yields

$$
\begin{aligned}
\dot{\boldsymbol{b}}= & -\operatorname{curl}(\eta \operatorname{curl} \boldsymbol{b}) \\
& -\operatorname{curl}\left(\alpha\left(\operatorname{curl} \boldsymbol{B}_{0} \times \boldsymbol{b}+\operatorname{curl} \boldsymbol{b} \times \boldsymbol{B}_{0}\right)\right) \\
\operatorname{div} \boldsymbol{b}= & 0
\end{aligned}
$$

describing the evolution of a small magnetic perturbation $\boldsymbol{b}$. For conditions as realised in the crusts of cooling isolated neutron stars, unstably growing perturbations $\boldsymbol{b}=\hat{\boldsymbol{b}}(\boldsymbol{r}) \exp \gamma t, \gamma>0$, can exist, with typical growth times $\gamma^{-1} \sim 10^{4 \ldots 5}$ years.

The existence of this instability has been shown in a slab geometry, approximating the neutron star crust geometry locally. Typical crustal density profiles were adopted. Boundary conditions are defined by assuming a perfect conductor being adjacent to the slab at the bottom and vacuum being adjacent at the top. That is, corresponding to the superconductivity of the NS core, the magnetic and electric fields do not penetrate under the bottom of the slab whereas the magnetic field is continued above the slab as a potential field approximating the conditions outside the NS.

${ }^{1}$ A surface region with a magnetic flux prevailingly normal to the surface and with a certain degree of axisymmetry.
The background fields $\boldsymbol{B}_{0}$ we used are parallel to the slab; they vary strongly with depth and vanish at the bottom of the slab. Fields of that kind, especially toroidal ones, may exist as relics of a short, but very efficient convective dynamo phase acting in the proto-neutron star (Thompson \& Duncan 1993; Urpin \& Gil 2003). However, it is perhaps more likely that such background fields are due to the action of a thermoelectric instability, which amplifies toroidal seed fields very effectively and sets on compulsory when $T_{\mathrm{s} 6}^{4} / g_{\mathrm{s} 14} \gtrsim 100$ (Wiebicke \& Geppert $1996, T_{\mathrm{s} 6}-$ surface temperature in $10^{6} \mathrm{~K}, g_{\mathrm{s} 14}$ - surface gravity in $10^{14} \mathrm{~cm} \mathrm{~s}^{-2}$ ). Such conditions are met in almost all newly born neutron stars and are maintained up to an age of $\sim 1000$ years. Moreover, when a strong temperature gradient is established again by any external or internal process, the thermoelectric instability will be switched on again. It can thus, at later stages too, produce a toroidal background field which is capable of providing the conditions for the onset of the HDI. Hence, the initial strength of the background field is determined essentially either by the vigour of the dynamo or by the strength of the temperature gradient and may well exceed $10^{14} \mathrm{G}$ locally inside the crust.

Of course, in any real situation the background field is not exactly parallel to the slab: Within the region we are interested in, the polar cap, a normal background field arises quite naturally. Its influence on the properties of the HDI can at the moment only be extrapolated from the special case of a homogeneous normal background field component in a homogeneous slab. Then, the tangential part of the background field rotates about a normal axis with an angular velocity proportional to the normal field component. The HDI continues to exist if the ratio of normal to tangential background field components is not too large. If the normal field is small, what we want to assume here, the background configuration rotates slowly and we expect no major differences in the perturbation modes in comparison with those obtained for completely tangential background fields to appear.

The HDI is described in detail for conditions realised in the crusts of isolated cooling neutron stars in RKG03 and we refer the reader to that extended presentation. Its main result is that the HDI occurs for a variety of realistic crust conditions and that the obtained growth times are short enough to cause observable consequences.

The easiest way to get an idea on how the HDI acts is considering its analogy to field generation by hydrodynamic dynamo action. One major ingredient of dynamo models, e.g., those explaining the solar magnetic field, is shear motion (in axisymmetry typically occurring as differential rotation) which is capable of generating strong toroidal (in axisymmetry: azimuthal) fields from weak poloidal (in axisymmetry: meridional) fields by "winding up". In our context, the motion of the electron fluid, in which the field is partially frozen in, is well able to provide shear (see the term $\alpha \operatorname{curl} \boldsymbol{B}_{0} \times \boldsymbol{b}$ in Eq. (2)). To get a successful dynamo, shear has to be completed by another effect generating in turn poloidal fields from toroidal ones. Mean-field dynamo theory has identified various possibilities for that, amongst which one (the so-called $\omega \times \boldsymbol{j}$ effect) has exactly the same mathematical structure like the second Hall term $\alpha$ curl $\boldsymbol{b} \times \boldsymbol{B}_{0}$ in Eq. (2) (see, e.g., Rädler 1969). Thus, 


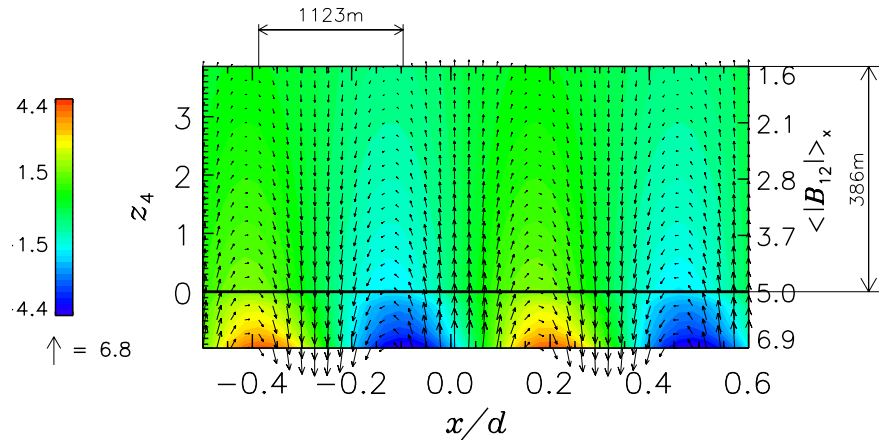

Fig. 1. Structure of a typical magnetic field perturbation generated by the Hall-drift induced instability from a toroidal crustal background field having a maximum field strength of about $10^{14} \mathrm{G}$. The growth time of the perturbation is of the order of $5 \times 10^{4}$ years. The neutron star model behind relies on the Pandharipande-Smith equation of state, its crustal thickness $d \sim 3800 \mathrm{~m}$. The vertical scale $z$ is arbitrarily limited to $z=d / 10=386 \mathrm{~m}$, where the magnetic field strength is reduced by a factor of about 3 compared with its surface value. The distance between adjacent spots in $x$-direction $\sim 1.1 \mathrm{~km}$, in $y$-direction $\sim 3 \mathrm{~km}$ (see Fig. 2 for that dimension). Colour encoding corresponds to the normal field component, $\langle\cdot\rangle_{x}$ denotes the average with respect to $x$. All magnetic field values are given in units of $10^{12} \mathrm{G}, z_{4}$ is in units of $10^{4} \mathrm{~cm}$.

a cycle of mutual amplification of poloidal and toroidal fields can establish resulting in a growing total field.

\section{The small-scale poloidal field}

The structure of a typical ${ }^{2}$ unstable (i.e., exponentially growing) perturbation field (or eigenmode) is shown in Figs. 1 and 2 (for detailed explanations see RKG03). While the background fields used in that paper are derived from the equatorial regions of global dipolar fields, we nevertheless apply here the corresponding results in the vicinity of the polar cap region. There, a global poloidal field can no longer provide a suitable background field structure - a toroidal field is needed. Applicability of the results of RKG03 then requires that the toroidal background field near the pole and the poloidal one near the equator may have more or less similar radial profiles. (Note, that the properties of the HDI modes are only moderately sensitive with respect to the radial profile.) This seems to be at least possible in the case of convective-dynamo generated fields and even a reasonable assumption for thermoelectrically generated fields with not too low multipolarity. For a maximum strength of the toroidal background field in the order of about $10^{14} \mathrm{G}$ the perturbation rises with a characteristic growth time of about $5 \times 10^{4}$ years. We assume, that at saturation it may reach a significant fraction of the background field's strength. Then it is possible that its surface strength reaches $5 \times 10^{12} \mathrm{G}$ and we scaled the perturbation field (the amplitude of which is not determined by the linear Eq. (2)) simply to this value.

The lifetime of such a perturbation is bounded from above by its ohmic decay time. Very likely, it is shorter because the

\footnotetext{
${ }^{2}$ Pandharipande-Smith equation of state, initial penetration density $\rho_{0}=10^{13} \mathrm{~g} \mathrm{~cm}^{-3}$, cubic background field $\boldsymbol{B}_{0}$ with initial polar field strength $10^{14} \mathrm{G}$, model age $3 \times 10^{5}$ years.
}

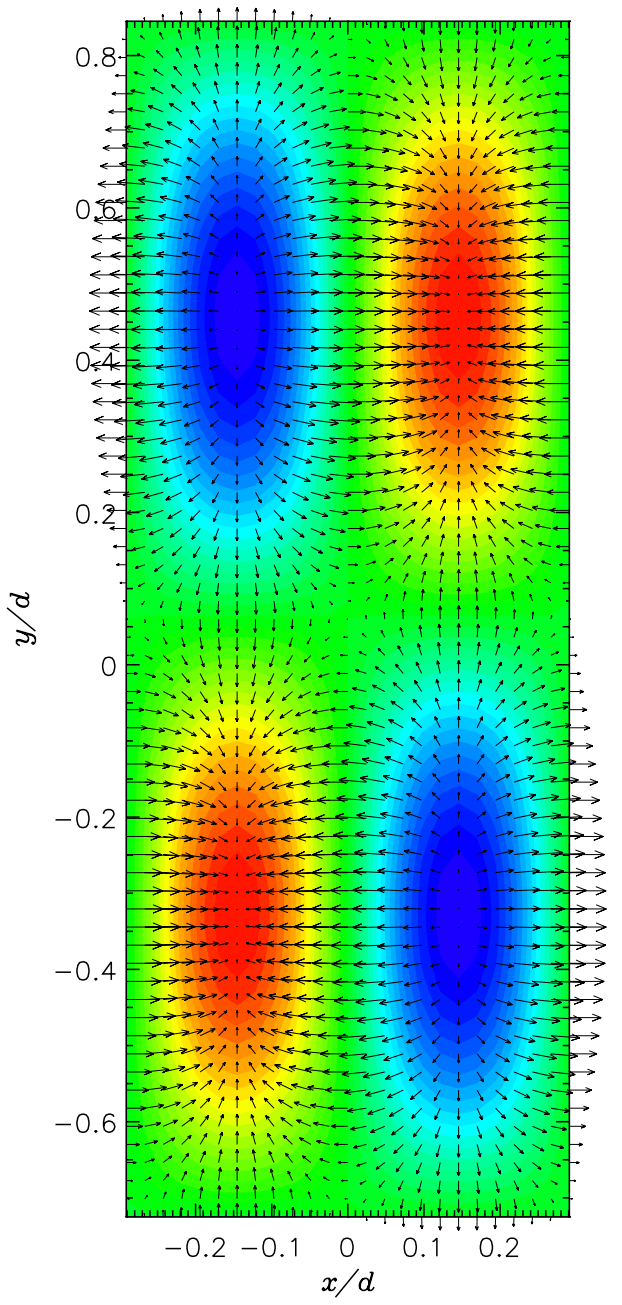

1.

0.0

$-1.7$

$-3.3$

$-5.0$

$\uparrow=4.7$

Fig. 2. The same magnetic field perturbation as in Fig. 1 but viewed from above and zoomed into a smaller $x$ interval. Note, that this field has to be superimposed on the global dipolar field having its pole in the vicinity of one of the spot centers to get the actual open magnetic field lines.

perturbation is subject to the Hall-effect which is supposed to accelerate the decay in general. With the conditions at the depth in which the perturbation currents circulate and their scales, we estimate the ohmic decay time of the field presented in Figs. 1 and 2 as $\gtrsim 10^{7}$ yrs.

Both the major radial and tangential scales of the unstable perturbations are given approximately by the radial extent of the background field. According to the assumptions about the latter they are significant portions of the crust thickness (say $\$ 50 \%$ ) and scale with it. The crust thickness, in turn, is model dependent: Stiff equations of state (EOS) result in smaller compactnesses and hence larger star radii and crust thicknesses in comparison with softer ones. For example, the specific EOSs considered in RKG03, the stiff PandharipandeSmith and medium soft Friedman-Pandharipande ones yield 
a crust thickness of $\sim 3.8 \mathrm{~km}$ and $700 \mathrm{~m}$, respectively. Generalising from these two cases we suggest that the scales of the eigenmodes are not very sensitive with respect to the star model.

Let us stress that the spot-like structure presented in Figs. 1 and 2 satisfies all conditions to create the vacuum gap and to generate the electron-positron plasma within it. In fact, Gil \& Mitra (2001) and Gil \& Melikidze (2002) argued that the vacuum gap can only be formed under the so-called near-threshold conditions, when the surface magnetic field $B_{\mathrm{s}} \gtrsim 0.1 B_{\mathrm{q}} \sim$ $4.4 \times 10^{12} \mathrm{G}$. In addition, it requires small radii of curvature $R_{\mathrm{c}} \ll 10^{6} \mathrm{~cm}$. As can be seen from Fig. 1, in our case $R_{\mathrm{c}}<10^{5} \mathrm{~cm}$. In such a strong and curved surface magnetic field the magnetic pair creation via curvature and/or ICS (inverse Compton scattering) photons is very efficient.

Moreover, the magnetic field lines of the perturbation field converge at local poles (spot centers) as can be seen in Fig. 2. As already mentioned at the end of Sect. 1, this property of the surface magnetic field is crucial for the subpulse drift phenomenon. Of course, as an implicit assumption one has to keep in mind that just one spot structure plays the role of a local magnetic pole requiring that the canonical polar cap (i.e., the one formed by the dipolar field alone) coincides at least partially with this spot. The actual polar cap is defined by the open field lines of the superimposed global star-centered-dipole and local perturbation fields, along which an ultra-high accelerating potential drop can exist, or, in other words, by those perturbation field lines which merge with the open dipolar field lines before entering the radio emission region (see Gil et al. 2002, for details).

When assuming that close to the surface the global dipolar field is at least five times smaller than the perturbation field the superposition of both fields would yield a field very similar to the one shown where only the symmetry between the regions with positive and negative normal flux is slightly disturbed.

Note, that the spot-like structures of the surface field must not be confused with the Deshpande-Rankin "hot spots" or "sparks" (Deshpande \& Rankin 1999). The former can be considered stationary within all the time scales relevant for observations and provide just the conditions for the development of sparks. By virtue of the $\boldsymbol{E} \times \boldsymbol{B}$ plasma drift the latter move around the local magnetic pole. As the appearance of the pure $\boldsymbol{E} \times \boldsymbol{B}$ drift is confined to homogeneous fields only, additional drift components and/or acceleration prevent the charge carriers forming the sparks from moving exactly periodically along closed paths. So it remains to be examined whether our field spots are suited to reproduce the periodicities of the subpulse phenomenon in detail.

Acknowledgements. This paper is supported in part by Polish grant 2P03D00819. J.G. acknowledges the renewal of the Alexander von Humboldt fellowship. U.G. and M.R. are grateful to the Arbeitsamt Berlin for financial support and to the AIP for hospitality.

\section{References}

Becker, W., Swartz, D. A., Pavlov, G. G., et al. 2003, ApJ, 594, 798 Deshpande, A., \& Rankin, J. 1999, ApJ, 524, 1008

Deshpande, A., \& Rankin, J. 2001, MNRAS, 322, 438

Fan, G. L., Cheng, K. S., \& Manchester, R. N. 2001, ApJ, 557, 297

Geppert, U., \& Rheinhardt, M. 2002, A\&A, 392, 1015

Gil, J., \& Melikidze, G. 2002, ApJ, 577, 909

Gil, J., Melikidze, G., \& Geppert, U. 2003, A\&A, 407, 315

Gil, J., Melikidze, G., \& Mitra, D. 2002, A\&A, 388, 235

Gil, J., \& Mitra, D. 2001, ApJ, 550, 383

Gil, J., \& Sendyk, M. 2000, ApJ, 541, 351

Gil, J., \& Sendyk, M. 2003, ApJ, 585, 453

Goldreich, P., \& Julian, H. 1969, ApJ, 157, 869

Goldreich, P., \& Reisenegger, A. 1992, ApJ, 395, 250

Haberl, F., Schwope, A., Hambaryan, V., Hasinger, G., \& Motch, C. 2003, A\&A, 403, L19

Hollerbach, R., \& Rüdiger, G. 2002, MNRAS, 337, 216

Pavlov, G. G., Zavlin, V. E., Sanwal, D., \& Trümper, J. 2002, ApJ, 569, L95

Rädler, K.-H. 1969, Monatsber. Dt. Akad. Wiss., 11, 194

Rheinhardt, M., \& Geppert, U. 2002, Phys. Rev. Lett., 88, 101103

Rheinhardt, M., Konenkov, D., \& Geppert, U. 2003, A\&A, submitted [astro-ph/0310917]

Ruderman, M., \& Sutherland, P. 1975, ApJ, 196, 51

Sanwal, D., Pavlov, G. G., Zavlin, V. E., \& Teter, M. A. 2002, ApJ, 574, L61

Shalybkov, D., \& Urpin, V. 1997, A\&A, 321, 685

Thompson, C., \& Duncan, R. 1993, ApJ, 408, 194

Urpin, V., \& Gil, J. 2003, A\&A, submitted

Vainshtein, S., Chitre, S., \& Olinto, A. 2000, Phys. Rev. E, 61, 4422

Wiebicke, H.-J., \& Geppert, U. 1996, A\&A, 309, 203 